\title{
Improvement of active power sharing ratio of $P / V$ droop controllers in low-voltage islanded microgrids
}

\author{
T. L. Vandoorn, J. D. M. De Kooning, B. Meersman and L. Vandevelde \\ Dept. of Electrical Energy, Systems \& Automation, Ghent University, \\ St-Pietersnieuwstraat 41, 9000 Gent, Belgium, e-mail: Tine.Vandoorn@UGent.be.
}

\begin{abstract}
Microgrids provide a coordinated integration of distributed generation units in the electrical power system. By operating in islanded mode, they can increase the reliability of the system or electrify remote areas. For the power sharing and voltage control in low-voltage microgrids, active power/grid voltage droop control is highly suitable. In order to optimize the integration of renewable energy sources in the microgrid, a variant of this droop control, the voltage-based droop (VBD) control, has been presented. A well-known concern about droop controllers is the inherent trade-off between voltage control and power sharing. Therefore, in this paper, an additional control loop is included in the VBD control to improve the active power sharing ratio. In this way, accurate power sharing is achieved, i.e., the DG units respond to load changes exactly according to their droops. Although this modification relies on communication, it does not jeopardize the reliability of the microgrid as if the communication is lost, the basic VBD control still ensures a stable microgrid operation, while operating without the need for communication.
\end{abstract}

Index Terms-distributed generation, droop control, microgrid, power sharing accuracy

\section{INTRODUCTION}

The strong increase of distributed generation (DG) units has a significant impact on the operation of the distribution networks. The networks are increasingly being confronted with congestion and voltage problems. Therefore, the current fitand-forget strategy of integrating DG in the network is not a sustainable option concerning the reliability of the power systems. A more coordinated approach is required, which can be provided by integrating DG, loads and storage elements into microgrids. Microgrids have a single point of connection with the distribution network, which enables them to operate independently in the islanded mode or stay connected to the distribution network in the grid-connected operation condition [1]. Microgrids are likely to play a key role in the evolution of the smart grid [2]. In this sense, the smart grid can emerge as a system of integrated smart microgrids [3].

This papers focusses on the islanded operation of the microgrid. In the islanded mode, the microgrid is independently responsible for both voltage and power control. As most microgrid elements are connected to the network through inverters, new control strategies for these inverters have been developed. Mirrored with conventional grid control, the droop control strategy has been implemented in microgrids. Both active power/frequency $(P / f)[4],[5]$ and active power/voltage $(P / V)[5]-[7]$ droops have been discussed. The latter copes with the mainly resistive lines in the low-voltage microgrids that are considered here. The voltage-based droop (VBD) control strategy of $[\overline{8}]$ extends the $P / V$ droops to microgrids with a high share of renewables.

The droop controllers ensure a stable microgrid operation, which is their key objective. However, sometimes, accurate power sharing needs to be guaranteed, thus, irrespective of the line parameters. In this way, a fair contribution of DG units in the microgrid control can be achieved. A disadvantage of droop control is that there is always a trade-off between the voltage control and the accuracy of the power sharing. The accuracy of power sharing or power sharing ratio reflects the contribution of each unit to cope with load variations compared to the other units. Perfectly accurate power sharing is achieved when the load variations are picked up by the DG units $(\Delta P)$ exactly according to their droops $(K)$. These droops are dependent on the ratings of the units and the controllability of the energy source. For example, gasfired power stations contribute more to the primary control to cope with load variations than nuclear power plants. This is analogous for DG units, where fully-controllable DG units contribute to the power sharing proportionally with their rated power, while less controllable units (such as many renewables) will contribute less.

The grid voltage $V$ is a local parameter and can be different in different network locations, which can affect the power sharing ratio. Therefore, in the $P / f-Q / V$ droop control, the reactive power $(Q)$ sharing ratio may differ from the droop ratio, i.e. inaccurate reactive power sharing. Similarly, the active power sharing ratio can be inaccurate in the $P / V$ $Q / f$ droop controllers. Several solutions to increase the power sharing accuracy have been presented in literature, focussing on the $P / f-Q / V$ droop controllers. In [9], a small highfrequency signal is injected in the system as control signal for the output active and reactive power. However, the circuitry required to measure the small real power variations in this signal adds to the complexity of the control [10]. In [10], each unit regulates its terminal voltage based on the reference voltage that is obtained from, firstly, the conventional $Q / V$ droops and, secondly, a correction term based on the measured load voltage. An analogous method to achieve accurate power sharing by introducing load voltage feedback is presented in [11].

In this paper, the method of [10] is modified to improve the active power sharing ratio in low-voltage networks. The modifications are twofold. Firstly, [10] focusses on $Q / V$ droops in the conventional $P / f-Q / V$ droop control, while here, the $P / V$ droops in the VBD control are adapted. Secondly, in microgrids, there is not a single load voltage. The loads and DG units are distributed in the network and the line impedances in between cannot be neglected. Hence, this paper suggests to communicate the active power output of the units instead of the load voltage to achieve accurate power sharing.

In $\S$ II the power sharing of the $Q / V$ and VBD controllers are analysed. The power sharing ratio is improved in $\S$ III This is achieved by, firstly, communicating the load voltage analogously to [10], and secondly, by communicating output 
active power measurements. In $\S[\mathrm{IV}$, a test feeder is studied in order to show the improvement of the power sharing ratio by using this active power correction in a dynamical situation with multiple DG units, VBD control and various loads.

\section{Power Sharing of CONVENTIONAL CONTROLleRs}

\section{A. Accuracy of $Q / V$ droops in the $P / f$ droop control method}

Droop control was introduced for standalone microgrid control in [4], [5]. The $P / f$ droop control method is based on mimicking the operation of synchronous generators. The $P / f$ droops of a unit $i$ follow

$$
\omega_{i}=\omega_{\text {ref }}-K_{\mathrm{f}, \mathrm{P}}\left(P_{i}-P_{\mathrm{i}, \mathrm{ref}}\right)
$$

with $K_{\mathrm{f}, \mathrm{P}}$ the droop, $\omega=2 \pi f, P_{i}$ the delivered power of unit $i$ and $P_{\mathrm{i}, \text { ref }}$ its reference power. Similarly, the amplitude $V_{\mathrm{g}, \mathrm{i}}$ of the grid voltage of this unit is drooped with the measured reactive power $Q_{i}$ :

$$
V_{\mathrm{g}, \mathrm{i}}=V_{\mathrm{g}, \text { ref }}-K_{\mathrm{f}, \mathrm{Q}}\left(Q_{i}-Q_{\mathrm{i}, \mathrm{ref}}\right)
$$

with $V_{\mathrm{g}, \mathrm{ref}}$ the nominal voltage amplitude and $Q_{\mathrm{i}, \mathrm{ref}}$ the reference reactive power of unit $i$. The droops are coordinated to make each DG system supplying active and reactive power in proportion to its power capacity. For the $P / f$ droops, accurate power sharing between each two units $i=1,2$ is always obtained:

$$
\frac{\Delta P_{1}}{\Delta P_{2}}=\frac{K_{\mathrm{f}, \mathrm{P}, 2}}{K_{\mathrm{f}, \mathrm{P}, 1}}
$$

with $\Delta P_{i}=P_{i}-P_{\mathrm{i}, \text { ref }}$, because the frequency is equal everywhere in the network. The latter is not valid for the terminal voltages $V$ of the DG units, which can differ due to different line impedances. This can affect the accuracy of the reactive power sharing. Hence, the reactive power sharing is only accurate if $\frac{\Delta Q_{1}}{\Delta Q_{2}}$ equals $\frac{K_{\mathrm{f}, \mathrm{Q}, 2}}{K_{\mathrm{f}, Q, 1}}$. To improve the reactive power sharing ratio, in [10], the $Q / V$ droop controller is changed. Each unit regulates its terminal voltage based on, firstly, the reference voltage $V_{\mathrm{i}}$ that is determined by the $Q / V$ droop and, secondly, the measured load voltage $V_{l}$. A correction voltage $V_{\mathrm{i} \text {,corr }}$ is determined

$$
V_{\mathrm{i}, \text { corr }}=K_{\mathrm{f}, \text { corr }} \int_{0}^{t}\left(V_{\mathrm{g}, \mathrm{i}}-V_{l}\right) d t
$$

with $V_{l}$ the communicated load voltage magnitude and $K_{\mathrm{f} \text {,corr }}$ a correction factor. Hence, the terminal voltage magnitude of the DG unit is controlled to $V_{\mathrm{i}, \text { corr }}$, whereas with conventional $Q / V$ droops, it is controlled to $V_{\mathrm{g}, \mathrm{i}}$. In this way, two DG units can share the reactive load accurately. The integrator gain $K_{\mathrm{f} \text {,corr }}$ can be varied to achieve the desired speed of response [10].

\section{B. Accuracy of $P / V$ droops in the $P / V$ droop control method}

1) $P / V$ droop control: While the $P / f$ droop control method works well in a microgrid with mainly inductive line impedances, it leads to a concern when implemented on a low-voltage microgrid, without significant inertia and where the line resistance should not be neglected [12]. In case of mainly resistive lines, the active power is mainly linked with the voltage difference, while reactive power is mainly linked with the phase angle, hence frequency. This leads to $P / V$

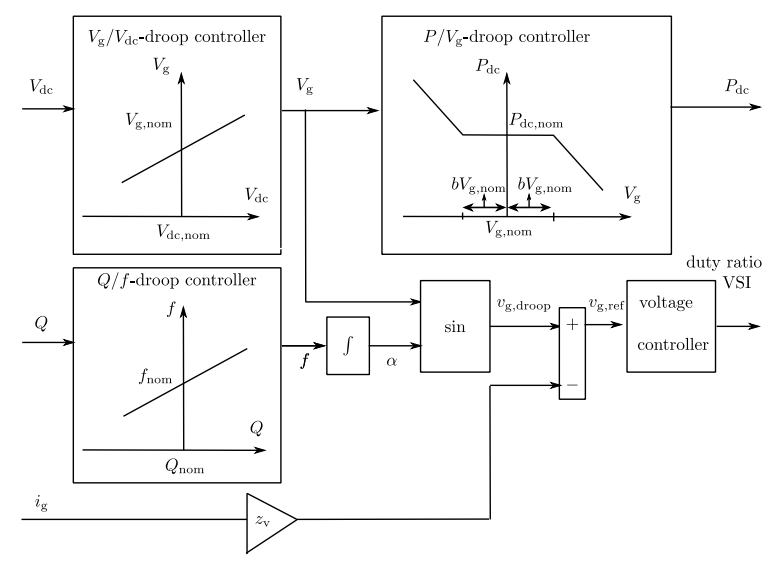

Fig. 1. VBD controller and virtual impedance loop

and $Q / f$ droops as opposed to the conventional $P / f$ and $Q / V$ droops $[5]-[7]$. The active and reactive power are measured and drooped to obtain the rms voltage and its frequency $(\omega=2 \pi f)$ respectively:

$$
\begin{gathered}
V_{\mathrm{g}, \mathrm{i}}=V_{\mathrm{g}, \mathrm{ref}}-K_{\mathrm{P}}\left(P_{i}-P_{\mathrm{i}, \mathrm{ref}}\right) \\
\omega_{i}=\omega_{\text {ref }}+K_{\mathrm{Q}}\left(Q_{i}-Q_{\mathrm{i}, \mathrm{ref}}\right)
\end{gathered}
$$

with $K_{\mathrm{P}}$ and $K_{\mathrm{Q}}$ the droops. As voltage is not a global parameter, the accuracy of the active power sharing can be affected.

2) VBD control: The voltage-based droop (VBD) control of [8] is a variant of $P / V$ droop control that focusses on the optimal integration of renewables in the network and also incorporates a dc-bus controller. In the VBD control, the $P / V$ droop controller is divided into two droop controllers and constant-power bands are included as depicted in Fig. 1 Further details of the control loops are given in [8].

The parameter $2 b$ determines the width of the constantpower band. In case the terminal voltage of the DG unit is in the constant-power band, i.e., $(1-b) V_{\mathrm{g}, \text { ref }}<V_{\mathrm{g}}<(1+$ b) $V_{\mathrm{g}, \text { ref }}$, the DG unit delivers its reference power. Otherwise, the power of the DG unit is changed. A distinction is made between dispatchable and less dispatchable DG units.

For dispatchable DG units, analogous as for the central large generators, $P_{\text {ref }}$ represents the scheduled power that can be determined in the electricity markets. In this case, the value of $b$ is small, such that the unit reacts on small variations of power to balance the load.

For less dispatchable DG units, such as many renewables, a wider constant-power band is used. In this way, these units do not react on all load variations but change their output power only in case of more extreme voltage variations compared to the dispatchable DG units. These extreme voltages occur merely when the power limits of the dispatchable DG units are nearly reached. Also, voltage is a local parameter, hence, the voltage limits can also be reached in areas where the ratio dispatchable versus less dispachtable DG units is high. The current fit-and-forget strategy of integrating DG, solves the voltage problems by turning the DG units off. Here, power curtailment is incorporated to capture more of the renewable energy. In this way, voltage problems can be overcome as the renewables also take part in the voltage control. An optimized 
integration of renewables is possible because the VBD control prioritizes the power changes of the units by setting different values of $b$ without the need for communication. In these units, $P_{\text {ref }}$ can, for example, represent the instantaneous maximum power point of a wind turbine.

As shown in Fig. 1, the $V_{\mathrm{g}} / V_{\mathrm{dc}}$ and $Q / f$ droop controllers, which are digital controllers, determine the droop voltage $v_{\mathrm{g}, \mathrm{droop}, \mathrm{k}}^{\star}=V_{\mathrm{g}, \mathrm{k}} \sin \left(\alpha_{\mathrm{k}}\right)$ ( $k$ is the discrete time instance). Discrete values are used because pulse width modulation with sampling period $T_{\mathrm{S}}$ is used in the converter. The amplitude $V_{\mathrm{g}}$ of the droop voltage is obtained from the $V_{\mathrm{g}} / V_{\mathrm{dc}}$ droop controller and the phase angle is obtained from the frequency $f$ in the $Q / f$ droop controller. Together, they determine

$$
v_{\mathrm{g}, \mathrm{droop}, \mathrm{k}}^{\star}=V_{\mathrm{g}, \mathrm{k}} \sin \left(\alpha_{\mathrm{k}-1}+2 \pi f_{\mathrm{k}} T_{s}\right)
$$

Fig. 11 also shows the virtual output impedance loop. A resistive output impedance $z_{v}=R_{v}$ is chosen as this provides more damping in the system [13] and complies with the power control strategies of the loads and generators, where the active power is changed based on the grid voltage:

$$
v_{\mathrm{g}}^{\star}=v_{\mathrm{g}, \mathrm{droop}}^{\star}-R_{v} i_{g}
$$

with $v_{\mathrm{g}}^{\star}$ the reference voltage for the voltage controller, $v_{\mathrm{g}, \text { droop }}^{\star}$ the voltage obtained by the $\mathrm{VBD}$ controller and $i_{g}$ the grid current.

Both the conventional $P / V$ and the VBD droop controller use voltage as trigger for active power changes. As voltage is not a global parameter and the line impedances are not neglected, accurate active power sharing is not guaranteed. This is analogous with the $Q / V$ droops, where the accuracy of reactive power sharing is affected.

\section{IMPROVED ACTIVE POWER SHARING}

In this section, first, the method of [10], which is developed for $Q / V$ droops, is modified to comply with the $P / V$ droops in the VBD control. Accurate power sharing is achieved, but the load voltage needs to be communicated. As in microgrids, there is no common load voltage, in the second paragraph, the output power of the DG units is communicated. In this way, the VBD control is adapted to achieve accurate power sharing between the DG units in a microgrid.

\section{A. Load voltage amplitude communication}

1) Control principle: In [10], the accuracy in power sharing of the $Q / V$ droop controller is improved by communicating the load voltage magnitude $V_{l}$ to all DG units. Based on this technique, in this paragraph, a modification to the VBD control is made to make the active power sharing, as here $P / V$ droops are used, more accurate and less dependent on the line impedances.

In order to improve the accuracy of the active power sharing, the reference voltage $v_{\mathrm{g} \text {,droop }}$ is modified in this paragraph. In (9), instead of using the voltage amplitude $V_{\mathrm{g}}$, which is the output of the $V_{g} / V_{\mathrm{dc}}$ droop controller, the reference voltage is calculated by using a correction voltage $V_{\mathrm{g}, \mathrm{corr}}$ :

$$
v_{\mathrm{g}, \mathrm{droop}, \mathrm{k}}^{\star}=V_{\mathrm{g}, \mathrm{corr}, \mathrm{k}} \sin \left(\alpha_{\mathrm{k}-1}+2 \pi f_{\mathrm{k}} T_{s}\right)
$$

The correction voltage is obtained from a measurement of the load voltage $V_{\mathrm{l}}$, which is communicated to the DG units, and the output $V_{\mathrm{g}}$ of the $V_{\mathrm{g}} / V_{\mathrm{dc}}$ droop controller:

$$
V_{\mathrm{g}, \mathrm{corr}}=K_{\mathrm{corr}} \int_{0}^{t}\left(V_{\mathrm{g}}-V_{\mathrm{l}}\right) \mathrm{dt}
$$

analogously as in (4). In discrete time (z-domain) for time instance $k$ :

$$
V_{\mathrm{g}, \mathrm{corr}, \mathrm{k}}=K_{\mathrm{corr}} \frac{T_{\mathrm{s}}}{z-1}\left(V_{\mathrm{g}, \mathrm{k}}-V_{\mathrm{l}, \mathrm{k}}\right)+V_{\mathrm{g}, \mathrm{ref}}
$$

The control scheme is summarized in Fig. 2

The VBD control strategy is a primary controller, focussing on the reliability of the microgrid, and hence, does not use communication. By including the correction voltage, communication is used. However, this does not jeopardize the reliability of the system. If the communication of $V_{l}$ fails, the controller falls back to the core VBD control, thus, without voltage correction. This core controller achieves a stable operation, but, the active power sharing can deviate from its pre-determined value. The communication of $V_{1}$ does not require a high bandwidth as only the amplitude of the signal needs to be communicated and not the instantaneous value.

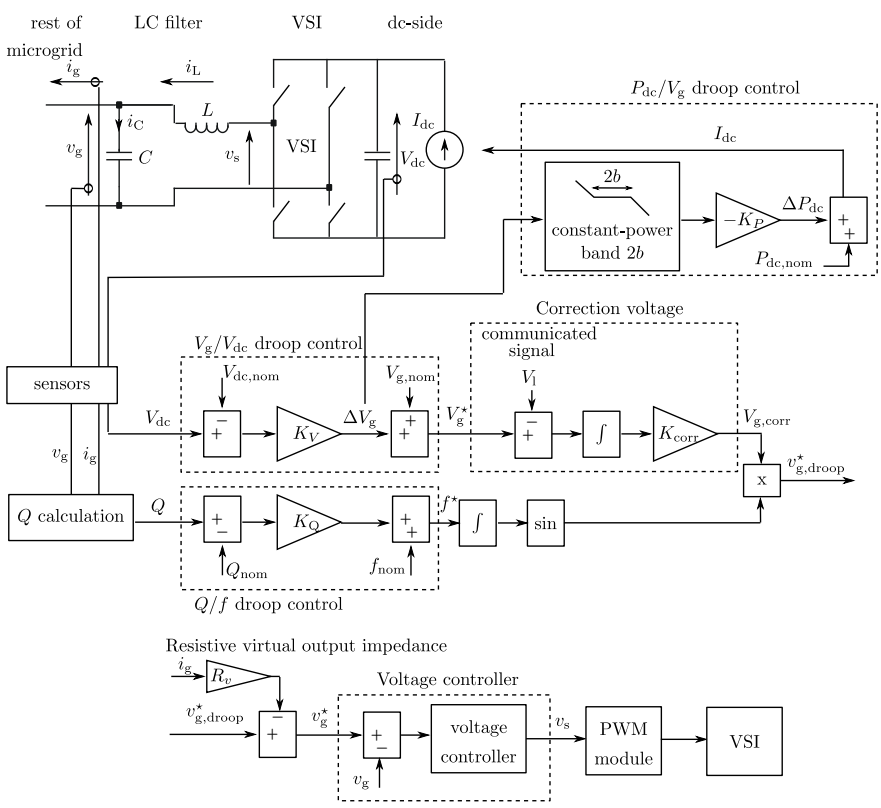

Fig. 2. VBD control with voltage correction and virtual output impedance

2) Example: The correction voltage is used to achieve a better active power sharing ratio in the simple microgrid of Fig. 3. This microgrid consists of two DG units and one load. A resistive virtual output impedance $R_{\mathrm{v}}=3 \Omega$ is included. The following parameters are used: $L=2 \mathrm{mH}, C=3 \mu \mathrm{F}$, $C_{\mathrm{dc}}=1.5 \mathrm{mF}, V_{\mathrm{dc}, \text { ref }}=450 \mathrm{~V}, V_{\mathrm{g}, \text { ref }}=230 \sqrt{2} \mathrm{~V}, f_{\mathrm{nom}}=$ $50 \mathrm{~Hz}, R_{1,1}=1 \Omega, R_{1,2}=0.3 \Omega, R=25 \Omega$ in case of a resistive load $(R$-load) or $P=2500 W$ for a constantpower load ( $P$-load), $b=0 \%, P_{\text {ref, } 1}=2000 \mathrm{~W}$ and $P_{\text {ref }, 2}=$ $1000 \mathrm{~W}$. The droops are $K_{\mathrm{V}}=0.5 \mathrm{~V} / \mathrm{V}$ for the $V_{\mathrm{g}} / V_{\mathrm{dc}}$ droop controller, $K_{\mathrm{p}, 1}=P_{\text {ref }, 1} / 50 \mathrm{~W} / \mathrm{V}, K_{\mathrm{p}, 2}=P_{\text {ref }, 2} / 50 \mathrm{~W} / \mathrm{V}$ for the $P / V_{\mathrm{g}}$ droop controller and $K_{\mathrm{Q}}=1 \cdot 10^{-4} \mathrm{~Hz} / \mathrm{VAr}$ for the $Q / f$ droop controller. When voltage correction $V_{\mathrm{g}, \mathrm{corr}, \mathrm{k}}$ is used, $K_{\text {corr }}$ equals 50 and $T_{\mathrm{s}}=50 \mu \mathrm{s}$.

Without voltage correction, accurate power sharing, i.e., $\Delta P_{1} / \Delta P_{2}=P_{\text {ref }, 1} / P_{\text {ref }, 2}=2$ would only be achieved when 
$P_{\text {ref, } 1} / P_{\text {ref, } 2}$ is equal to $R_{1,2} / R_{1,1}$, which is obviously not the case. In Table I, the cases with and without $V_{l}$ correction (i.e., using $V_{\mathrm{g} \text {,corr }}$ instead of $V_{\mathrm{g}}$ to determine the terminal grid voltage of the converter) are compared for a constantpower and resistive load. From the obtained simulation results, it follows that perfect power sharing is obtained when communicating the load voltage to the DG units and using it for the control in a correction voltage. Without $V_{l}$ correction, the power sharing is affected by the line impedance values.

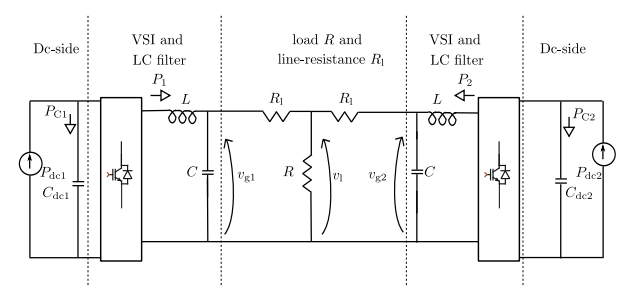

Fig. 3. Simple microgrid configuration: two DG units and a resistive load

TABLE I

VOLTAGE CORRECTION : LOAD VOLTAGE AMPLITUdE $\left(V_{1}\right)$ COMMUNICATION

\begin{tabular}{c||c|c|c} 
case & $P_{1}(\mathrm{~W})$ & $P_{2}(\mathrm{~W})$ & $\Delta P_{1} / \Delta P_{2}$ \\
\hline no $V_{1}$ correction, $R$-load & 1257 & 879 & 6.1 \\
$V_{1}$ correction, $R$-load & 1546 & 775 & $\mathbf{2 . 0}$ \\
\hline no $V_{1}$ correction, $P$-load & 1502 & 1060 & -8.3 \\
$V_{1}$ correction, $P$-load & 1710 & 854 & $\mathbf{2 . 0}$
\end{tabular}

\section{B. Output active power communication}

1) Control principle: An issue with the communication of $V_{l}$ as used in the previous paragraph and [10], is that it conflicts with the extended microgrid configurations. Microgrids consist of feeders with different loads and DG units connected to them, at different places. The line impedances cannot be neglected in these cases. Subsequently, a general $V_{l}$ does not exist in the microgrid. Therefore, in this paragraph, the active power output of the DG units is communicated to the other units and a method is presented to adapt the VBD control to achieve accurate power sharing.

When using active power communication, the power sharing accuracy can be enforced directly. In this case, the correction voltage for DG unit $i$ at instance $k$ is obtained by using

$$
V_{\mathrm{g}, \mathrm{corr}, \mathrm{k}, \mathrm{i}}=K_{\mathrm{corr}} \frac{T_{\mathrm{s}}}{z-1}\left(\frac{P_{\text {ref }, \mathrm{i}}}{P_{\text {ref }, \mathrm{j}}}-\frac{P_{\mathrm{k}, \mathrm{i}}}{P_{\mathrm{k}, \mathrm{j}}}\right)+V_{\mathrm{g}, \mathrm{k}, \mathrm{i}}
$$

with $P_{\mathrm{k}, \mathrm{j}}$ the communicated output active power of another unit $j$ and $V_{\mathrm{g}, \mathrm{k}, \mathrm{i}}$ the output of the $V_{\mathrm{g}} / V_{\mathrm{dc}}$ droop controller of unit $i$ at instance $k$.

2) Example: The same case as in the previous paragraph is studied with $K_{\text {corr }}=200$. The obtained results are depicted in Table III. It follows that by using $P$ correction, perfect power sharing is achieved as well. For three DG units, the control strategy remains the same. In the next paragraph, a more extended microgrid with multiple DG units is studied.
TABLE II

ACTIVE POWER COMMUNICATION

\begin{tabular}{c||c|c|c} 
case & $P_{1}(\mathrm{~W})$ & $P_{2}(\mathrm{~W})$ & $\Delta P_{1} / \Delta P_{2}$ \\
\hline no $P$ correction, $R$-load & 1257 & 879 & 6.1 \\
$P$ correction, $R$-load & 1440 & 720 & $\mathbf{2 . 0}$ \\
\hline no $P$ correction, $P$-load & 1502 & 1060 & -8.3 \\
$P$ correction, $P$-load & 1715 & 857 & $\mathbf{2 . 0}$
\end{tabular}

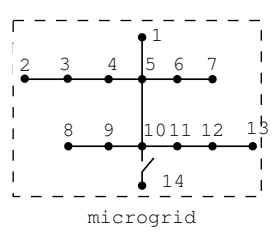

Fig. 4. IEEE 13 Node Test Feeder

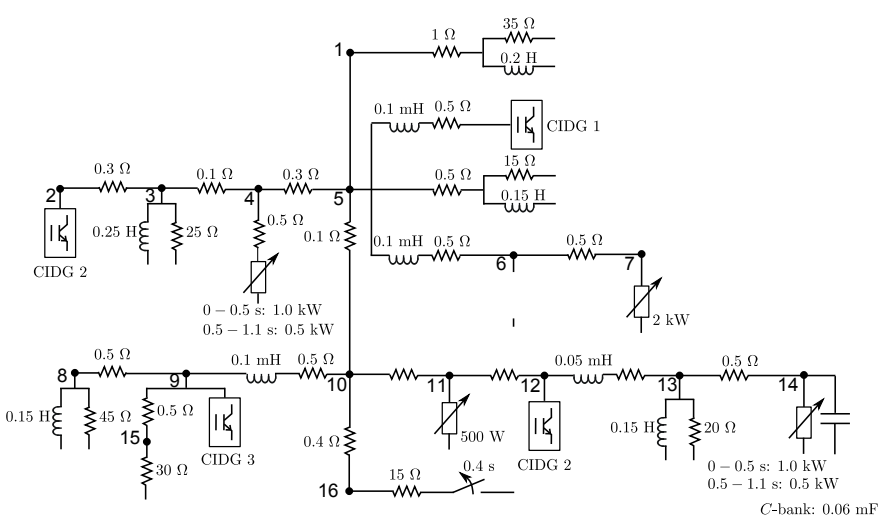

Fig. 5. Considered Test Feeder

\section{CASE STUDY}

A test feeder inspired by the IEEE 13 Bus Test Feeder as shown in Fig. 4 is simulated. The same basic configuration as the IEEE feeder is used, but with modifications for application as a low-voltage network in islanded mode, e.g., a higher $\mathrm{R} / \mathrm{X}$ value of the lines. This chosen feeder is interesting to study the effect of different load types, load changes and DG unit output variations in a small but realistic microgrid. The details of the nodes are summarized in Fig. 5, showing that a combination of various loads (resistive, inductive, constant-power and switching loads) is used. There are four converter-interfaced DG (CIDG) units connected to the feeder, with parameters summarized in Table III. The units use a resistive virtual output impedance $z_{\mathrm{v}}=3 \Omega$. A dynamical load and DG unit profile is included as the output of DG 3 and that of some loads vary in time (see Fig. 5).

The simulation results in Fig. 6, for VBD control with $P$ correction, show a stable microgrid operation. The decreased output power of CIDG3 at $t=0.5 \mathrm{~s}$ is clearly picked up by the other two DG units. Also, the load decrease at node 16 at $t=0.4 \mathrm{~s}$ and at nodes 4 and 14 at $t=0.5 \mathrm{~s}$ lead to acceptable transients that are mitigated fast by the VBD controllers.

When comparing the cases with and without $P$ control, on the one hand, both controls strategies achieve a stable microgrid operation, which is the main function of primary control. On the other hand, by including $P$ control, accurate 


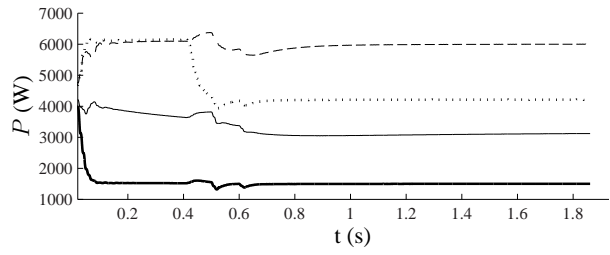

Fig. 6. Test feeder: with communication of $P(-=\mathrm{DG} 1 ;----=\mathrm{DG} 2, \cdots$ = DG $3 ;-=$ DG4 ): output power $P$

power sharing according to the droops is achieved as shown in Table IV

For the sharing between three units, the same case is studied but with $b=0 \%$ in DG4, such that this becomes a dispatchable DG unit in Table $\mathrm{V}$ It is concluded that the accuracy of power sharing is improved by using $P$ communication and a $P$ correction term in the voltage controller.

TABLE III

CIDG UNITS IN TEST FEEDER: PARAMETERS

\begin{tabular}{c||c|c} 
CIDG & $P_{\text {ref }}$ & $b$ \\
\hline DG1 & $3.15 \mathrm{~kW}$ & $0 \%$ \\
DG2 & $6 \mathrm{~kW}$ & $0 \%$ \\
DG3 & $t<0.3 \mathrm{~s}: 6.3 \mathrm{~kW}$ & $8 \%$ \\
& $t>0.3 \mathrm{~s}: 4.2 \mathrm{~kW}$ & \\
DG4 & $1.5 \mathrm{~kW}$ & $10 \%$
\end{tabular}

TABLE IV

SIMULATION RESULTS EXTENDED MICROGRID

\begin{tabular}{c||c|c|c} 
DG unit & $P_{\text {ref }}$ & no $P$ corr & $P$ corr \\
\hline DG1 & $3.15 \mathrm{~kW}$ & $3.8 \mathrm{~kW}$ & $3.1 \mathrm{~kW}$ \\
DG2 & $6.0 \mathrm{~kW}$ & $5.0 \mathrm{~kW}$ & $6.0 \mathrm{~kW}$ \\
DG3 & $4.2 \mathrm{~kW}$ & $4.2 \mathrm{~kW}$ & $4.2 \mathrm{~kW}$ \\
DG4 $(b=10 \%)$ & $1.5 \mathrm{~kW}$ & $1.5 \mathrm{~kW}$ & $1.5 \mathrm{~kW}$ \\
$P_{1} / P_{2}$ & 0.52 & 0.77 & $\mathbf{0 . 5 2}$ \\
$P_{1} / P_{4}$ & 2.10 & 2.55 & $\mathbf{2 . 1 0}$
\end{tabular}

TABLE V

SIMULATION RESULTS EXTENDED MICROGRID: OTHER $b$ FOR DG 4

\begin{tabular}{c||c|c|c} 
DG unit & $P_{\text {ref }}$ & no $P$ corr & $P$ corr \\
\hline DG1 & $3.15 \mathrm{~kW}$ & $3.64 \mathrm{~kW}$ & $3.65 \mathrm{~kW}$ \\
DG2 & $6.0 \mathrm{~kW}$ & $4.80 \mathrm{~kW}$ & $6.94 \mathrm{~kW}$ \\
DG3 & $4.2 \mathrm{~kW}$ & $4.20 \mathrm{~kW}$ & $4.20 \mathrm{~kW}$ \\
DG4 $(b=0 \%)$ & $1.5 \mathrm{~kW}$ & $2.52 \mathrm{~kW}$ & $1.74 \mathrm{~kW}$ \\
$P_{1} / P_{2}$ & 0.52 & 0.76 & $\mathbf{0 . 5 2}$ \\
$P_{1} / P_{4}$ & 2.10 & 1.44 & $\mathbf{2 . 1 0}$ \\
$\Delta P_{1} / \Delta P_{2}$ & & -0.41 & $\mathbf{0 . 5 2}$ \\
$\Delta P_{1} / \Delta P_{4}$ & & 0.48 & $\mathbf{2 . 0 4}$
\end{tabular}

\section{Conclusion}

In this paper, the power sharing ratio of $P / V$ droop control in general and VBD control in specific is improved through a correction term that uses communication. With VBD control, a stable microgrid operation is obtained but the power sharing is not perfectly accurate. Possibly, some DG units are slightly more burdened to cope with load variations than others, which can cause discussions concerning fairness. Therefore, an additional control loop is included to achieve accurate power sharing. A power correction term is calculated in each dispatchable DG unit by using the communicated signals of the DG units' output power. The output of the $V_{\mathrm{g}} / V_{\mathrm{dc}}$ droop controller together with this power correction term determines the reference grid voltage amplitude. This paper shows that in this way, a perfect power sharing ratio, i.e., according to the droops, is obtained. If the communication fails, the control strategy falls back to the core VBD controller achieving a stable operation, which is the aim of primary control, and a reasonably good power sharing ratio.

\section{ACKNOWLEDGEMENTS}

The work of $\mathrm{T}$. Vandoorn is financially supported by a Fellowship of the FWO-Vlaanderen (Research Foundation Flanders, Belgium). This research has been carried out in the frame of the Interuniversity Attraction Poles Programme initiated by the Belgian Science Policy Office (IAP-VII-02) The research of J. D. M. De Kooning is funded by the Special Research Fund (BOF) of Ghent University (Belgium).

\section{REFERENCES}

[1] R. H. Lasseter, A. Akhil, C. Marnay, J. Stephens, J. Dagle, R. Guttromson, A. Meliopoulous, R. Yinger, and J. Eto, "The CERTS microgrid concept, white paper on integration of distributed energy resources," in California Energy Commission, Office of Power Technologies - U.S. Department of Energy, LBNL-50829, http://certs.lbl.gov, Apr. 2002.

[2] N. Lidula and A. D. Rajapakse, "Microgrids research: A review of experimental microgrids and test systems," Renewable and Sustainable Energy Reviews, vol. 15, no. 1, pp. 186-202, 2011.

[3] H. Farhangi, "The path of the smart grid," in IEEE Power \& Energy Magazine, Jan./Feb. 2010, vol. 8, no. 1, pp. 18-28.

[4] R. H. Lasseter and P. Paigi, "Microgrid: A conceptual solution," in Proc. IEEE Power Electron. Spec. Conf. (PESC 2004), Aachen, Germany, 2004.

[5] A. Engler, O. Osika, M. Barnes, and N. Hatziargyriou, DB2 Evaluation of the local controller strategies. www.microgrids.eu/micro2000, Jan. 2005.

[6] A. Engler and N. Soultanis, "Droop control in LV-grids," in Proc. Internat. Conf. On Future Power Systems, The Netherlands, Nov. 16$18,2005$.

[7] C. Sao and P. Lehn, "Control and power management of converter fed microgrids," vol. 23, no. 3, Aug. 2008.

[8] T. L. Vandoorn, B. Meersman, L. Degroote, B. Renders, and L. Vandevelde, "A control strategy for islanded microgrids with dc-link voltage control," vol. 26, no. 2, pp. 703-713, Apr. 2011.

[9] A. Tuladhar, H. Jin, T. Unger, and K. Mauch, "Control of parallel inverters in distributed AC power systems with consideration of line impedance effect," vol. 36, no. 1, pp. 131-138, Jan./Feb. 2000.

[10] C. K. Sao and P. W. Lehn, "Autonomous load sharing of voltage source converters," vol. 20, no. 2, pp. 1009-1016, Apr. 2005.

[11] Q.-C. Zhong, "Robust droop controller for accurate proportional load sharing among inverters operated in parallel," vol. 58, 2011, to appear.

[12] Y. W. Li and C. N. Kao, "An accurate power control strategy for power electronics interfaced distributed generation units operating in a low voltage multibus microgrid,” vol. 24, no. 12, pp. 2977-2988, Dec. 2009.

[13] W. Yao, M. Chen, J. M. Guerrero, and Z.-M. Qian, "Design and analysis of the droop control method for parallel inverters considering the impact of the complex impedance on the power sharing," vol. 58, no. 2, pp. 576-588, Feb. 2011. 